\title{
eJRIEPS
}

Ejournal de la recherche sur l'intervention en éducation physique et sport

$30 \mid 2013$

Varia

\section{"L'activité » des élèves en risque de décrochage scolaire en EPS : la dynamique des interactions en classe}

Jacques Méard

\section{(2) OpenEdition}

Édition électronique

URL : http://journals.openedition.org/ejrieps/2492

DOI : $10.4000 /$ ejrieps.2492

ISSN : 2105-0821

Éditeur

ELLIADD

Référence électronique

Jacques Méard, « «L'activité » des élèves en risque de décrochage scolaire en EPS : la dynamique des interactions en classe », eJRIEPS [En ligne], 30 | 2013, mis en ligne le 01 juillet 2013, consulté le 08 octobre 2019. URL : http://journals.openedition.org/ejrieps/2492 ; DOI : 10.4000/ejrieps.2492

La revue eJRIEPS est mise à disposition selon les termes de la Creative Commons Attribution 4.0 International License. 
eJRIEPS 30 juillet 2013

"L'activité » des élèves en risque de décrochage scolaire en EPS :

la dynamique des interactions en classe

Jacques Méard

LAMHESS, EA 6309, Université Nice Sophia Antipolis

\section{Introduction}

Le décrochage scolaire est le principal indicateur de la réussite et de l'échec des politiques d'éducation (Commission Européenne, 2000). II se traduit par l'abandon prématuré du parcours scolaire, souvent à l'issue d'un désengagement progressif, un "décrochage de l'intérieur » (Bonnery, 2003) : de l'ennui, des distractions épisodiques puis fréquentes, des échecs cumulés ou mal acceptés conduisant à un désinvestissement croissant et des absences. Au-delà des facteurs de risques attribués aux élèves, plusieurs auteurs soulignent que cette dynamique se développe dans les interactions d'enseignementapprentissage (Blaya, 2010).

Le phénomène est souvent rapporté aux mauvais résultats de l'élève mais aussi au " sens " qu'il attribue ou non au travail scolaire. Celui-ci est énoncé sous forme de règles sociales (être à l'heure, ne parler qu'après autorisation, ne pas se moquer des camarades, etc.) et disciplinaires (les contenus scolaires prennent aussi le format de règles énoncées en classe) (Méard, Bertone \& Flavier, 2008). L'adolescent leur donne un sens, s'y soumet sans comprendre, les négocie ou les enfreint selon une dynamique difficilement prévisible. Les situations d'enseignement constituent des lieux de transaction à propos de ces règles (Woods, 1978 ; Strauss, 1990 ; Coulon, 1991 ; Bertone \& al., 2002). Dans ce processus, les interactions entre pairs peuvent avoir des effets sur l'intégration, le dégoût ou le réengagement scolaire chez des élèves à risque de décrochage : amitiés, hostilités, harcèlement (Brown, 2010) : effet positif, en termes d'aide pour des élèves en difficulté, mais, dans la plupart des études, impact négatif des moqueries (Thornberg, 2010b), ou simplement de la comparaison des élèves dans une classe (Thijs, Verkuyten \& Helmond, 2010) sur l'accrochage scolaire.

Pour comprendre la dynamique de co-construction de sens des règles du travail scolaire chez des élèves en risque de décrochage, en prenant en compte les interactions professeur-élèves, et entre élèves, nous recourons au modèle culturaliste de Leontiev (1981) et Engeström (2000) qui analysent l'évolution du « sens » en fonction de tensions 


\section{eJRIEPS 30 juillet 2013}

dans les systèmes d'activité (entre sujets, objets, outils, règles du travail, communauté et division du travail) (figure 1).

Figure 1. La structure de l'activité humaine (d'après Engeström, 2006)
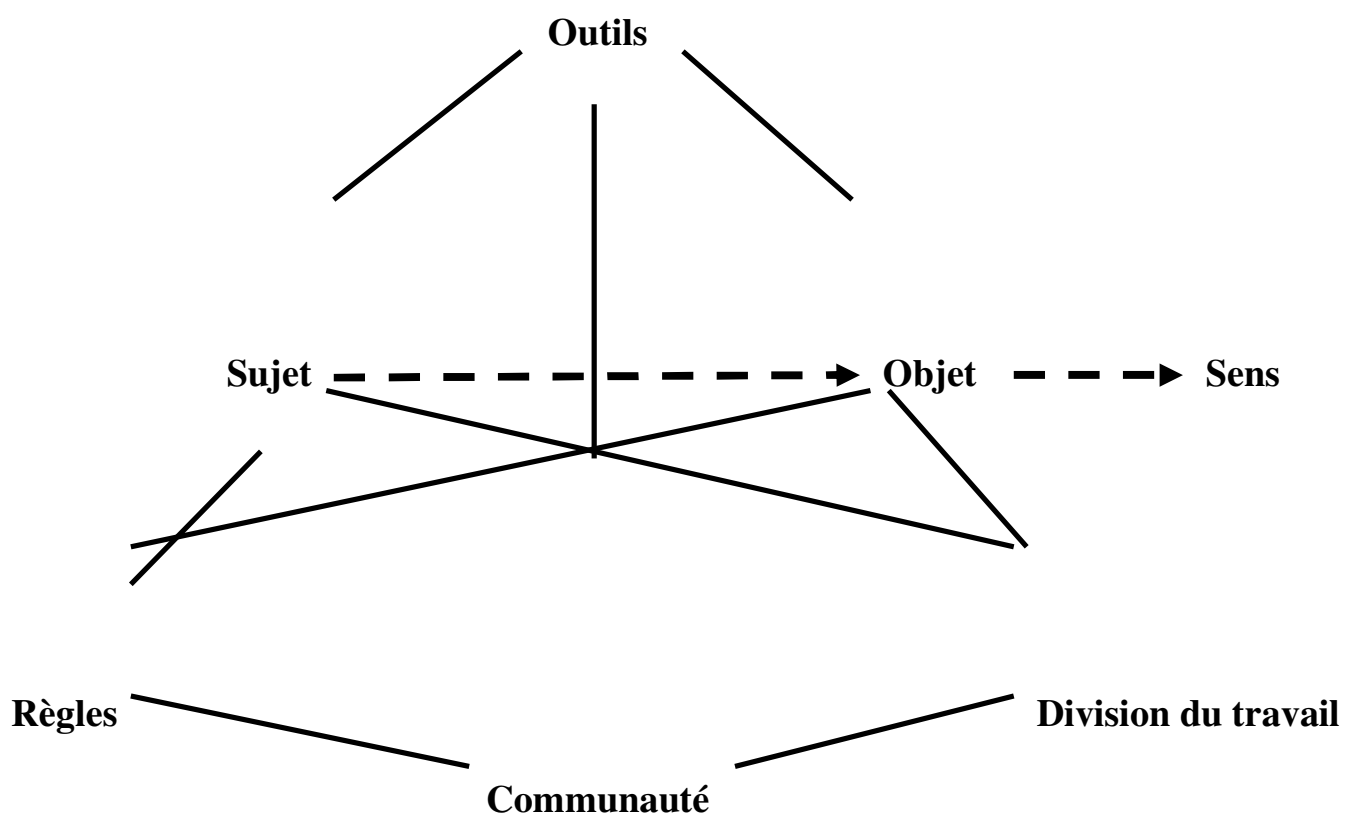

Selon ces auteurs, c'est dans la résolution de tensions émergeant des organisations qu'un nouveau sens est possible et que de nouvelles pratiques sont introduites.

\section{Méthode}

Dans un programme de recherche ${ }^{1}$ impliquant cinq collèges en France, nous présentons une étude d'un an avec trois enseignants expérimentés (mathématiques, technologie et EPS), et leur classe de troisième de 24 élèves de 14 à 16 ans (11 garçons and 13 filles) dont sept élèves (cinq garçons et deux filles) désignés «en risque de décrochage scolaire » par les enseignants.

Le recueil de données a consisté en de nombreuses données ethnographiques (62 heures - 42 leçons, dont 21 heures en EP (12 leçons) : interprétations à chaud, étayées par de courts entretiens enregistrés auprès des professeurs (36 entretiens) et des élèves (45 entretiens) juste après la leçon. Six leçons ont été analysées en profondeur: enregistrement vidéo, auto-confrontation avec l'enseignant concerné, interrogé à propos de ses actions en classe.

Le traitement des données s'est développé en 4 étapes :

\footnotetext{
${ }^{1}$ Soutenu par la « Fondation de France »
} 


\section{eJRIEPS 30 juillet 2013}

*d'abord le corpus entier des verbalisations au cours des six leçons a été divisé en unités de transactions. Une transaction a été définie comme la séquence d'interaction incluant (a) le même objet (c'est-à-dire soit une seule règle énoncée, soit un enchaînement de règles liées les unes aux autres (par exemple, tu dois rester au bord du terrain (parce que) tu dois être prêt à entrer dans l'équipe à la prochaine rotation) et (b) les mêmes interlocuteurs ;

*ensuite les règles énoncées dans chaque unité de transaction ont été codées de façon standardisée, en dissociant (a) les prescriptions-proscriptions ("tu dois", "tu ne dois pas"), qui pouvait prendre des formes nuancées ("ce serait mieux que tu ...", "essaie de...") ou présentées comme une question ("qu'est-ce que tu dois faire, là ?"); (b) les actions à faire ("faire ceci" "faire de cette façon"); et (c) les motifs ("pour", "parce que", "si tu ne fais pas ..."), qui étaient aussi parfois énoncées sous forme de questions ("pourquoi tu ne ferais pas de cette façon ?"). Par exemple, l'action "vous vous souvenez, pour réussir une rédaction, on définit d'abord les termes du sujet" fut codée "vous devez (a) / définir d'abord les termes du sujet (b) / pour réussir une rédaction (c)". L'action "Silence! Les autres classes travaillent à côté" fut codée "vous devez (a) / vous lever en silence (b) / pour laisser travailler les autres classes à côté (c)" ;

*de plus, les actions d'élèves furent observées (à partir des enregistrements) dans les unités de transaction et croisées avec les données d'entretiens pour mettre en évidence les dynamiques d'attribution de sens, en rapport avec les actions de l'enseignant (tableau de quatre volets, faisant succéder les unités de façon chronologique (Tableau 1). Volet 1 (actions et interactions verbales en classe), Volet 2 (règles codées), Volet 3 (entretien post-leçon avec l'enseignant) et Volet 4 (entretien avec l'élève) ;

"puis, chaque chercheur a interprété les leçons observées de façon indépendante en effectuant une triangulation des sources (observations, interactions verbales pendant des leçons et les entretiens) et en cherchant les tensions entre systèmes d'activité

*enfin, une triangulation des chercheurs a permis d'analyser les types de tensions, les types de dynamiques co-construction de sens, les scénarios-types en rapport avec le décrochage scolaire. Cette étape a abouti à des accords, la plupart du temps faciles à établir. En cas de désaccords, ils regardaient ensemble les éléments de la chronologie des transactions, les enregistrements des leçons et le discours de l'enseignant au cours de l'autoconfrontation. 


\section{eJRIEPS 30 juillet 2013}

\section{Résultats}

L'analyse des données pointe quatre tensions principales dans les systèmes d'activité analysés dans cette classe de troisième en éducation physique (Bob), mathématiques (Anne) et technologie (Eric). Ces tensions sont à la base des diverses formes de désengagement des élèves en risque de décrochage scolaire dans les systèmes d'activités.

\section{Les tensions « sujet-objet-division du travail »}

Dans les interactions en classe, un des éléments marquants concerne le caractère instable de l'engagement des élèves à risque de décrochage dans les tâches énoncées par l'enseignant. Ces engagements intermittents sont liés à la succession rapide de plusieurs motifs (ne pas se faire remarquer, retenir la leçon, entretenir des connivences avec certains camarades, avoir la meilleure note possible, etc.) et à la concurrence de ces motifs en fonction des circonstances.

Parmi ces motifs concurrents dans l'activité des élèves en risque de décrochage, certains sont attendus par l'enseignant, certains sont repérés mais inattendus, certains ne sont pas repérés. Le lien entre action et motif, autrement dit la relation sujet-objet est donc régulièrement source de désaccords, de malentendus qui provoquent des tensions dans le système d'activité.

\section{Les tensions « sujet-outils-objet»}

Dans cette dynamique d'engagements intermittents, les phases de décrochage analysées dans nos données sont aussi liées à un échec dans la réalisation de tâches. Un élève qui suit la règle du travail scolaire va à un moment se désengager de cette action parce qu'il ne peut résoudre un problème ou du fait de sa médiocre prestation. Dans ce cas l'impossibilité momentanée de réaliser ce que l'enseignant prescrit conduit à des changements de motifs (se reposer, discuter avec les copains). Le sens que l'élève attribue aux règles du travail scolaire n'est pas indépendant de son efficience (au sens de Leontiev). Le désengagement peut concerner également l'enseignant qui, à son tour, ne maîtrise pas les outils.

\section{Les tensions « sujet-objet-communauté 》}

Les effets de la maîtrise d'outils par l'élève sur le déplacement de l'objet de son activité touchent aussi le pôle « communauté » du système d'activité. Les interactions avec les autres élèves représentent parfois un moyen d'obtenir des ressources pour agir (en 


\section{eJRIEPS 30 juillet 2013}

apportant des « opérations »). Dans certains cas (par exemple, dans certains travaux de groupe), elles peuvent également fournir l'occasion à des élèves en risque de décrochage d'agir en fonction de motifs sociaux qui permettent de prolonger leur engagement dans une situation d'apprentissage. Les données d'entretiens auprès des élèves permettent de distinguer de possibles changements de motifs (je réponds à une question à haute voix pour aider la classe à comprendre la notion / pour m'assurer que j'ai compris la notion / pour briller devant mes camarades / pour interrompre le cours en déclenchant un moment festif) qui incite souvent l'élève à changer aussi d'actions (ne plus répondre à une question à haute voix).

\section{Les tensions sujet-règles-communauté}

Les dilemmes sont parfois forts chez un adolescent entre le motif qui est énoncé ou suggéré par l'enseignant et qui suscite parfois son adhésion et ce qui est suggéré par la « communauté » dont il ne veut sous aucun prétexte être exclu. Les tensions proviennent de la concurrence de motifs en fonction de règles énoncées par l'enseignant et le collectif de pairs. Ces règles du collectif d'élèves sont adressées à tous et ont la particularité de ne pas être explicites, contrairement à celles du travail scolaire. Ne pas montrer d'enthousiasme pour le travail scolaire, ne pas se mettre en avant, montrer à l'enseignant que l'on a son libre-arbitre, se moquer avec les autres d'une situation ou d'une action ridicule, il existe des impératifs auxquels l'élève ne peut pas déroger, sous peine de devenir une cible de moqueries.

\section{Discussion}

En EPS particulièrement, la plupart des élèves en risque de décrochage sont finalement engagés dans le travail, suivent les règles énoncées et attribuent à leurs actions des motifs attendus par l'enseignant (j'agis pour gagner la partie, réaliser une action difficile, faire progresser mon équipe). Le désengagement concerne en revanche plusieurs autres élèves (filles), non désignées comme en risque de décrochage, qui invoquaient régulièrement des inaptitudes (simulées) pour ne pas pratiquer. Or il semble que l'enseignant d'EPS manifeste moins de préoccupations pour ce type de décrochage-là.

Les tensions identifiées dans le système d'activité de cette classe au cours de l'année scolaire sont à l'origine de moments de désengagements qui participent au processus de décrochage des élèves «à risque ». C'est aussi dans la résolution de ces tensions 


\section{eJRIEPS 30 juillet 2013}

présentes au sein des organisations que de nouvelles pratiques sont introduites en EPS (Engeström, 2000).

\section{Bibliographie}

Bertone, S., Méard, J., Flavier, E., Euzet, JP. \& Durand, M. (2002). Undisciplined actions and teacher - student transactions during two physical education lessons. European Physical Education Review, 2(8), 4-21.

Blaya, C. (2010). Décrochages scolaires : l'école en difficulté. Bruxelles: De Boeck.

Bonnery, S. (2003). Le décrochage scolaire de l'intérieur : interaction de processus sociaux, cognitifs, subjectifs et langagiers. Les Sciences de l'éducation-Pour l'Ėre nouvelle, 36, 39-57.

Commission Européenne (2000). European report on quality of school education. Sixteen quality indicators. (Rapport)

Coulon, A. (1991). Ethnométhodologie et éducation. Paris : PUF.

Engeström, Y. (2000). Activity theory as a framework for analyzing and redesigning work. Ergonomics, 43(7), 960-974.

Leontiev AN (1981). Problems of the development of the mind. Moscow: Progress.

Méard, J., Bertone, S. \& Flavier, E. (2008). How fourth grade-students internalize rules during teacher-student(s) transactions. A case study. British Journal of Educational Psychology, 78, 395-410.

Thijs J, Verkuyten M and Helmond $P$ (2010). A further examination of the big-fish-littlepond effect: perceived position in class, class size, and gender comparisons. Sociology of Education, 83(4), 333-345.

Strauss, A. (1992). La trame de la négociation. Sociologie qualitative et interactionnisme. Paris : L'Harmattan.

Thornberg, R. (2010). School democratic meetings: Pupil control discourse in disguise. Teaching and Teacher Education, 26, 924-932.

Woods, P. (1978). Negotiating the demand of schoolwork. Curriculum Studies, 10 (4), 309-327. 